\title{
Electron Energy Loss Near Edge Structure (ELNES) on the Carbon K-Edge in Transition Metal Carbides with the Rock Salt Structure
}

\author{
Alan J. Craven and Laurence A.J. Garvie (*) \\ Department of Physics and Astronomy, University of Glasgow, Glasgow G12 8QQ, Scotland
}

(Received September 9; accepted December 22, 1994)

\begin{abstract}
The mono-carbides of the 3d, 4d and 5d transition metals of groups IVA (Ti, Zr, Hf) and $\mathrm{VA}(\mathrm{V}, \mathrm{Nb}, \mathrm{Ta})$ of the periodic table have the rock salt (B1) structure. They are-found in many technologically important systems. The binary compounds exist over a range of compositions, normally with a deficit of carbon. Since their structures are identical and their lattice parameters similar, there is considerable mutual solubility between then so that a wide range of compounds of the form $\mathrm{MC}_{x}$ exist where $\mathrm{M}$ is a mixture of transition metals. This paper presents a comparison of the electron energy loss near edge structure (ELNES) on the carbon K-edges from the binary carbides with compositions close to stoichiometry. The spectra show systematic changes of the ELNES and the threshold energy as the metal species is changed. The threshold energy and intensity increase on going from group IVA to group VA. Equivalent features can be identified in the spectrum from each compound. The energy of each feature, relative to the threshold energy, varies systematically with the lattice parameter of the compound. The systematic change in the ELNES with composition has potential applications where mixed metal carbides, nitrides and carbo-nitrides co-exist. In such cases, it can be difficult to obtain an unambiguous interpretation of microanalytical data and ELNES offers one more channel of information.
\end{abstract}

\section{Introduction}

The mono-carbides of the $3 \mathrm{~d}, 4 \mathrm{~d}$ and $5 \mathrm{~d}$ transition metals of groups IVA (Ti, $\mathrm{Zr}, \mathrm{Hf}$ ) and VA (V, $\mathrm{Nb}, \mathrm{Ta}$ ) of the periodic table have the rock salt (B1) structure. The binary compounds, $\mathrm{MC}_{x}$, exist over a range of compositions, normally with a deficit of carbon (i.e. $x \leq 1$ ). Since their structures are identical and their lattice parameters similar, there is considerable mutual solubility between them so that a wide range of compounds of the form $\mathrm{MC}_{x}$ also exist where $\mathrm{M}$ is now a mixture of transition metals.

Thus they provide a convenient group of compounds in which to study the effects on the shape of the electron energy loss near edge structure (ELNES) of the carbon K-edge resulting from

$\left({ }^{*}\right)$ Now at the Department of Geology, Arizona State University, Tempe, AZ-85287-1404, U.S.A. 
the systematic change of the metal species. Since such compounds are found in many technologically important systems (e.g. they are used to provide hardening in low alloy steels), there is considerable interest in their characterisation using microanalytical techniques. Study of the ELNES is one aspect of such characterisation. This information can be obtained with nanometre spatial resolution when electron energy loss spectroscopy with parallel detection (PEELS) is used in conjunction with a scanning transmission electron microscope (STEM) equipped with a field emission gun (FEG). Such a combination also offers high energy resolution combined with the good collection efficiency required to acquire spectra before radiation induced mass loss becomes significant [1].

This paper concentrates on the ELNES from binary MC carbides close to stoichiometry. Fink and co-workers have presented high energy resolution spectra from $\mathrm{TiC}$ [2], $\mathrm{VC} \mathrm{[3]} \mathrm{and} \mathrm{NbC}$ [4] recorded using a dedicated electron spectrometer with no imaging capability operated with an incident energy of $170 \mathrm{keV}$. Their spectrometer integrated over a range of scattering vectors, $q$, with a full width at half maximum (FWHM) of $0.2 \AA^{-1}$ whereas the spectrometer used for this work integrates over a range of $\sim 2 \AA^{-1}$ in order to provide both high spatial resolution and high collection efficiency. In general, the spectral shapes are in good agreement. The binding energies of the carbon 1s states in these compounds relative to that of carbon in hydrocarbon contamination have also been determined by Ramqvist et al. [5] using the technique of electron spectroscopy for chemical analysis (ESCA) (also known as X-ray photo-electron spectroscopy (XPS)). Their results have been converted into binding energies relative to the Fermi level and are quoted in reference [6]. There are significant variations in the threshold energies observed in the three sets of work.

\section{Experimental Method}

2.1 EQUIPMENT. - The spectra were recorded using a UHV version of the GATAN 666 parallel electron energy loss spectrometer controlled by the GATAN EL/P software. The spectrometer was mounted on a VG Microscopes HB5 FEGSTEM with post-specimen lenses and a z-lift stage. The HB5 was operated at an accelerating voltage of $100 \mathrm{kV}$. An additional interface has been inserted between the spectrometer and the GATAN bright field/dark field detectors so that an additional aperture mechanism can be fitted. This allows the entrance aperture of the spectrometer to be defined independently of the central aperture of the annular detector without interfering with the use of this detector for imaging.

The HB5 was used to form a probe of $\sim 1 \mathrm{~nm}$ in diameter containing a current of $\sim 0.2 \mathrm{nA}$ with a convergence semi-angle of $11 \mathrm{mrad}$. The post-specimen lenses were used to compress the electron distribution emerging from the specimen by a factor of $\sim 10$ so that a $1.25 \mathrm{~mm}$ diameter spectrometer entrance aperture accepted a semi-angle of $12.5 \mathrm{mrad}$ at the specimen. Under these conditions, it is possible to obtain a zero loss peak with a FWHM of $\sim 0.31 \mathrm{eV}$ in the asrecorded spectrum. If the current emitted from the tip is reduced by $\sim 1000$, the FWHM drops to $\sim 0.26 \mathrm{eV}$. Thus the performance of the system is similar to that reported by Krivanek $e t$ al. using an optically identical system [7]. Good energy resolution is also obtained in the core loss region of the spectrum using our system. On the carbon K-edge from graphite, the sharp peak at the onset of transitions to the $\sigma^{*}$ states is well resolved [8]. It has a FWHM of $\sim 0.3 \mathrm{eV}$ after de-convolving the point spread function of the detector. The system also resolves two features separated by $\sim 0.5 \mathrm{eV}$ on the $L_{3}$ edge of the $\mathrm{Mn}^{3+}$ ion in bixbyite [9].

Using the conditions described above, the intensity of the zero loss peak saturates the detector even with the shortest available integration time of $\sim 25 \mathrm{~ms}$. To limit the integration time further, GATAN provide an "attenuate" mode which sweeps the spectrum across the detector in a direction normal to dispersion direction once during each read-out cycle. In this way, the spec- 
trum is only on the sensitive area of the detector for $\sim 1 \mathrm{~ms}$ per read-out cycle, making this the effective integration time. Unfortunately the stability of the set-up for this mode is not very good. To avoid this mode and to provide a wide range of short effective integration times, the beam blanking system of the HB5 is used to limit the time the probe is incident on the specimen. This is implemented using hardware timing circuitry synchronised to the detector read-out cycle.

2.2 The Materials And Specimen Preparation. - The titanium carbide was prepared by arc-melting and was in the form of large-grained polycrystalline ingot whose composition was $\mathrm{TiC}_{0.98}$, as determined by gravimetric analysis. The other carbides were bought as commercial powders. The suppliers stated that they were stoichiometric and $99 \%$ pure but no detailed analyses were provided. A small amount of each compound was lightly crushed in an agate mortar and pestle. The crushed material was dispersed in a volatile organic liquid (either reagent quality acetone or propan-2-ol). After allowing the larger fragments to settle, drops of the dispersal were placed onto holey carbon films supported on copper specimen grids and the solvent allowed to evaporate.

2.3 RECORDING THE SPECTRA. - Spectra were recorded from thin flakes and edges overhanging the holes in the carbon support film. A spectrometer dispersion of $0.1 \mathrm{eV} / \mathrm{channel}$ was used. A single integration of between 2 and 8 seconds was used to record a spectrum in the region containing the carbon K-edge while a spectrum in the low loss region was recorded as the sum of 100 acquisitions each with a $40 \mathrm{msec}$ integration time during which the beam was un-blanked for $\sim 1$ msec. In this way both regions were recorded over similar times. All spectra were recorded from areas having a thickness of less than $0.25 \lambda_{\mathrm{T}}$, where $\lambda_{\mathrm{T}}$ is the inelastic mean free path.

The spectrometer dispersion and the energy offset between different spectral regions were obtained by applying known voltages to the isolated flight tube of the spectrometer using the GATAN voltage scan module (VSM). This voltage had previously been calibrated against the first peak of the $L_{3}$ edge of $\mathrm{NiO}$, as described in reference [10]. This calibration was checked during the experiments by measuring the energy of the $\pi^{*}$ peak at the onset of the carbon K-edge of graphite, using graphite particles found on the specimens. This energy was found to be $285.0 \pm 0.1 \mathrm{eV}$ in excellent agreement with that quoted by Fink [3].

For each area of the specimen examined, a number of spectra were recorded. Five or six Kedge spectra were recorded, each with a slightly different offset voltage. This allowed the effects of the channel to channel variations in the detector response to be minimised, as described in section 2.4. With the exception of the $5 \mathrm{~d}$ compounds, four pairs of spectra, each consisting of a low loss region and a K-edge region, were recorded to determine the threshold energy. For the $5 \mathrm{~d}$ compounds only one pair per area was recorded. Appropriate dark currents for the spectra were also recorded.

Since such a large number of spectra were recorded from a given area, the probe was scanned over a raster during the acquisitions to minimise the effects of radiation damage known to occur in such materials [1]. Only limited areas of thin material of relatively constant thickness were available and the size of the typical raster was $13 \mathrm{~nm} \times 10 \mathrm{~nm}$. The shapes of the initial spectra from a given area were always identical. In some of the carbides, changes in shape were seen in later spectra. If such changes were observed, the raster was moved to an adjacent area. Only spectra with the same shape as the first were retained in the analysis below.

2.4 Selection of the AREAS From Which the Spectra Were Recorded. - All areas of the $\mathrm{TiC}_{0.98}$ gave similar data. However, it rapidly became clear that the samples made from the commercial powders contained many small pieces of material which were far from stoichiometry as well as particles of free graphite, presumably un-reacted material from the original process, 
and pieces of agate from the mortar and pestle. The latter materials posed no problem and the graphite provided a useful calibration check, as noted above. To avoid the small sub-stoichiometric particles, spectra were taken only from the thin edges of rather large particles with well-defined straight outlines. Such material gave ELNES with sharper features than the sub-stoichiometric material.

In addition, if the un-apertured probe (containing a current of $\sim 10 \mathrm{nA}$ ) was stopped on a particle a few microns in size, then the temperature of the particle became very high. In many cases, the particle melted causing a reaction with the adjacent film or deposition of small crystals of the material onto the surrounding film. This was achieved with $\mathrm{ZrC}, \mathrm{HfC}, \mathrm{VC}$ and $\mathrm{TaC}$. At first sight, this behaviour may appear surprising, bearing in mind that these materials are refractory with melting points in the range of 3500 to $4500 \mathrm{~K}$. However, such particles had dimensions approaching the electron range (typically tens of microns) so that a large fraction of the incident electron energy was absorbed while the thin holey carbon support film minimised heat loss by conduction. Evidence of the high temperatures attained was provided by the resulting graphitisation of the surrounding amorphous carbon film.

Material produced in this way is likely to approach stoichiometry since it is formed from relatively large grains of the powder in the presence of excess carbon from the support film. As expected, the ionisation edges obtained from the reaction zone at the edge of the carbon film or from deposited crystals overhanging the support film showed ELNES similar to that obtained from the edges of large grains.

For $\mathrm{TiC}_{0.98}$, the data presented are from thin edges; for $\mathrm{VC}$, the data is from the reaction zone at the edge of the carbon film; for $\mathrm{ZrC}$ and $\mathrm{NbC}$, the data is from the edges of well-defined grains; for $\mathrm{HfC}$ and $\mathrm{TaC}$, the data is from re-deposited crystals. For each compound, the spectrum shown is the sum of spectra from several areas which gave similar sharp ELNES.

2.5 PROCESSING THE SPECTRA. - To obtain the edge shape, the dark current was first subtracted from each of the selected K-edge spectra, after which the background was subtracted, using an $A \cdot E^{-r}$ form where possible. In the cases of $\mathrm{ZrC}$ and $\mathrm{NbC}$, the preceding metal $M_{4,5}$ edge makes this difficult. In such cases, a straight line extrapolation was used. The carbon $\mathrm{K}$ edges from the individual spectra were then aligned and summed. The resulting spectrum was smoothed by averaging over a range of adjacent channels. The range of channels was chosen to be the largest that caused no appreciable broadening of the steepest part of the initial onset. Finally, the effect of the tailing caused by the point spread function of the detector was removed using the "sharpen resolution function" of the EL/P software. This effectively replaces the zero loss peak shape by a Gaussian peak of similar FWHM. No attempt was made to deconvolute the multiple scattering.

The threshold energy was determined by noting the energy difference between the positions of the zero-loss peak and the point half way up the steepest part of the edge onset and then adding the appropriate VSM voltage. This was done for each pair of spectra recorded for this purpose. In this way, several values were obtained from each of the areas for which data was included in the edge shape of the material. These values were then averaged to give the threshold energy.

\section{Results and Discussion}

The ELNES observed on the carbon K-edges is shown in Figures 1 and 2. In Figure 1, the upper three spectra compare the shapes of the group IVA compounds and the lower three spectra compare the group VA compounds. The spectra have been scaled so that they have the same areas in the region up to $325 \mathrm{eV}$ i.e. just prior to the $\mathrm{Zr} M_{3}$ edge threshold at $330 \mathrm{eV}$ (just visible in the 
$\mathrm{ZrC}$ spectra of Fig. 1). The edge shapes of $\mathrm{TiC}, \mathrm{VC}$ and $\mathrm{NbC}$ agree well with those in references [2-4]. The peaks and shoulders are labelled following Pflüger et al. [2]. In both groups IVA and $\mathrm{VA}$, it is clear that peak 1 is broadened and lowered relative to peaks 3 and 4 on going from the $3 \mathrm{~d}$ to the $5 \mathrm{~d}$ transition metals. The deep minimum present $\sim 8 \mathrm{eV}$ above the threshold in the $3 \mathrm{~d}$ compounds also becomes less significant. This is consistent with the band structure calculations of Neckel and co-workers; the band structure of TiC shows a band-gap at a similar energy above the Fermi level [11] whereas that for NbC shows no bandgap in this region [12].

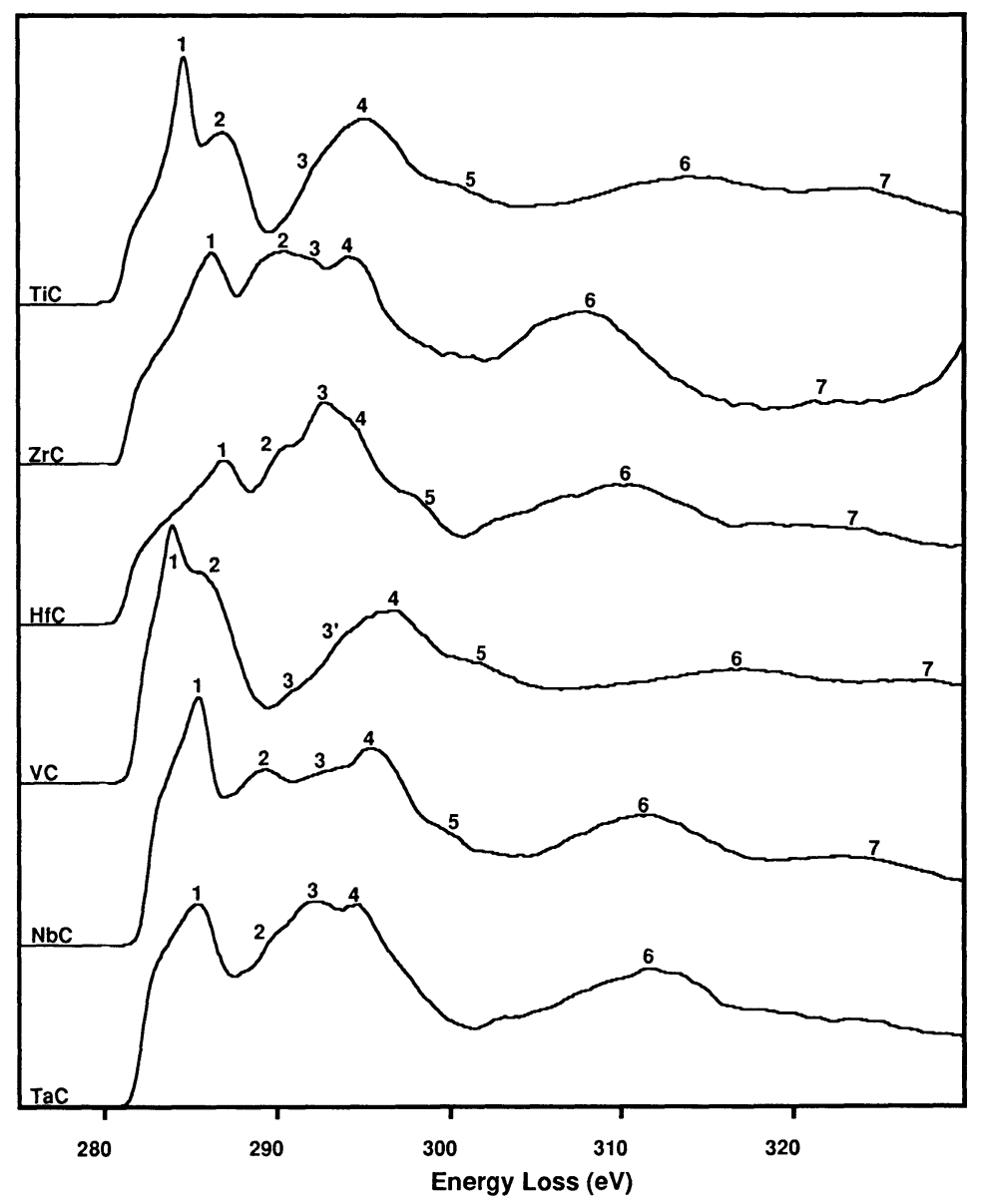

Fig. 1. - Comparison of the carbon K-edge shapes in the rock salt transition metal carbides of groups IVA and VA. The spectra are presented to facilitate comparison of the compounds within the same group. The integrated intensities are scaled to be the same in the energy range up to $325 \mathrm{eV}$.

In Figure 2, the spectra are shown with an expanded energy scale and reordered to facilitate comparison of compounds with metal species from the same transition series. For all three transition series, the energy difference between the threshold and peak 1 is greater in the group IVA compounds than in the equivalent group VA compounds. The absolute threshold energies and the intensity at the threshold of the group IVA compounds are lower than that those of the corre- 
sponding group VA compounds. These effects reflect the increased number of outer electrons in the group VA compounds. In addition, the absolute energies of peak 1 are higher and the absolute energies of peak 4 are lower in the group IVA compounds than in the equivalent group VA compounds. Thus peaks 1 to 4 occurs over a smaller energy range in the group IVA compound and this range lies within that of the corresponding group VA compound.

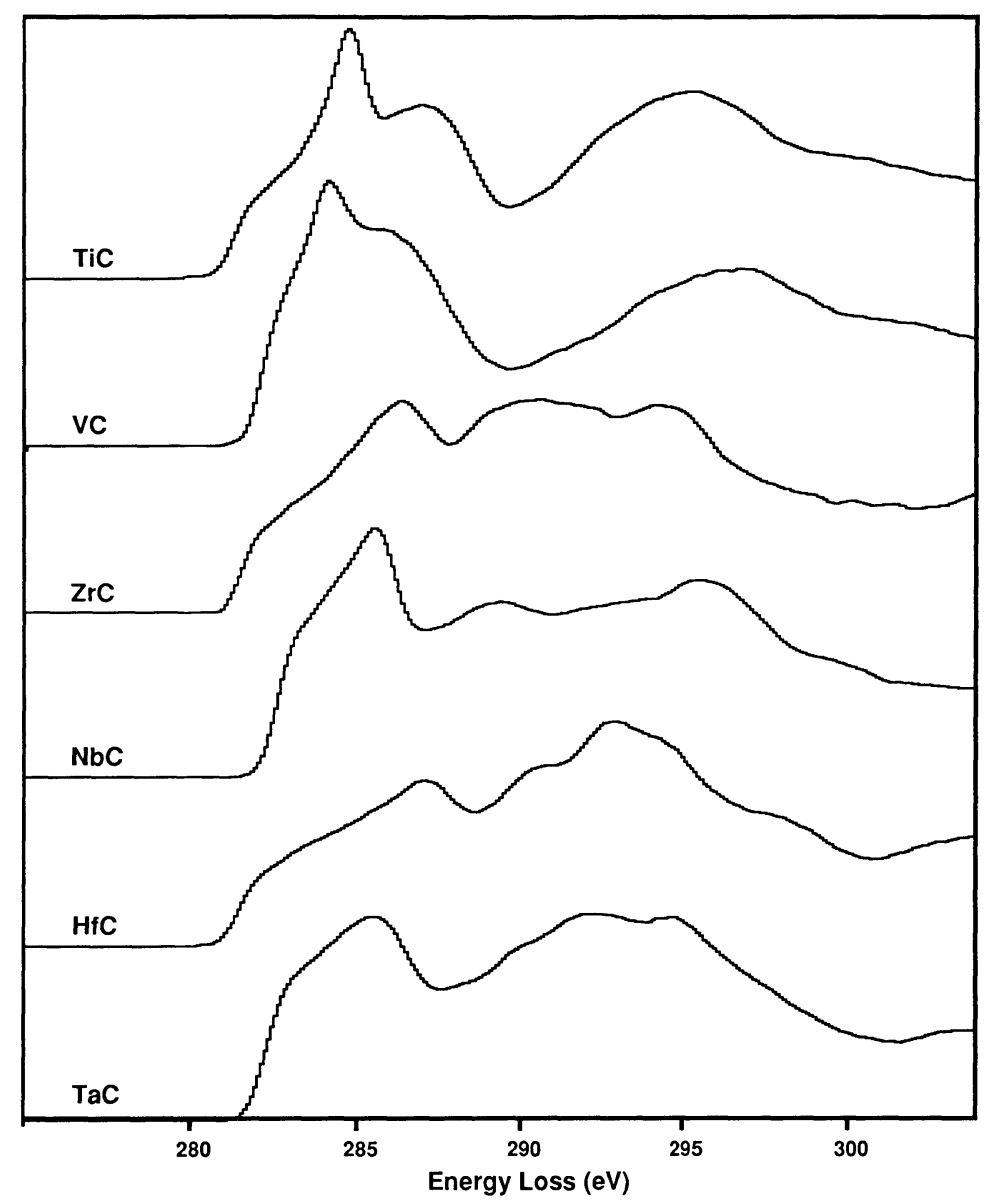

Fig. 2. - The same data as Figure 1 but shown with an expanded energy scale to emphasize the near threshold structure and in an order that facilitates comparison of compounds containing metals from the same transition series.

Turning to look at the numbered features in more detail, their energies relative to the threshold are given in Table I along with the observed threshold energies and the lattice parameters for the compounds, as given by Goldschmidt [13]. These lattice parameters are for those compounds whose compositions are closest to stoichiometry. The blanks in Table I correspond to the cases in which it was not possible to identify the appropriate feature. Some of the features are weak and so there is greater uncertainty in their energy. Such features are marked with an asterisk. In particular feature 3 is rather ill-defined in TiC and VC. Pflüger et al. [2] do not identify feature 3 in 
their TiC spectrum. However, there appears to be a weak shoulder on the low energy side of peak 4 on the TiC spectrum in Figure 2 and so this has tentatively been labelled feature 3 in Figure 1. The shoulder labelled 3 on the VC spectrum in Figure 1 is also weak. In the position marked, there is a strong feature in the VC spectrum of Fink [3]. However, without this supporting evidence, an alternative interpretation of the shape is possible and one could place the feature several $\mathrm{eV}$ higher at $3^{\prime}$.

Table I. -- The lattice parameter, a, the threshold energy and the energies of the numbered features above the threshold for each compound. The features whose energies are marked with an asterisk (*) are rather weak and the energy may not be exact.

\begin{tabular}{|c|c|c|c|c|c|c|c|c|c|}
\hline & $a$ & Threshold & 1 & 2 & 3 & 4 & 5 & 6 & 7 \\
\hline & $(\AA)$ & $(\mathrm{eV})$ & $(\mathrm{eV})$ & $(\mathrm{eV})$ & $(\mathrm{eV})$ & $(\mathrm{eV})$ & $(\mathrm{eV})$ & $(\mathrm{eV})$ & $(\mathrm{eV})$ \\
\hline $\mathrm{TiC}$ & 4.3280 & 281.2 & 3.5 & 5.7 & $10.8^{*}$ & 14.0 & 19.9 & 32.6 & 43.3 \\
\hline $\mathrm{ZrC}$ & 4.6983 & 281.4 & 4.9 & 9.0 & 10.7 & 12.6 & & 26.4 & $40.4^{*}$ \\
\hline $\mathrm{HfC}$ & 4.6430 & 281.4 & 5.5 & 8.8 & 11.3 & 12.8 & 16.9 & 28.2 & 41.8 \\
\hline $\mathrm{VC}$ & 4.1655 & 282.0 & 2.0 & 3.9 & $9^{*}$ & 14.3 & 20.3 & 34.6 & 45.9 \\
\hline $\mathrm{NbC}$ & 4.4707 & 282.5 & 3.0 & 6.8 & 10.1 & 13.0 & 17.5 & 28.9 & 42.2 \\
\hline $\mathrm{TaC}$ & 4.4555 & 282.2 & 3.2 & 7.4 & 9.8 & 12.3 & & 29.8 & \\
\hline
\end{tabular}

It is clear that the energy of a feature relative to the threshold does not vary monotonically in going from the $3 \mathrm{~d}$ to the $5 \mathrm{~d}$ transition series e.g. feature 6 . Such features are often the result of resonances in the backscattering of the ejected electron by the nearest neighbours [14]. Thus it is likely that the peak energy is related to the lattice parameter of the compound rather than the atomic number of the metal species, as predicted by Natoli [15]. The condition for such a resonance is that the backscattered atomic electron returns to the original site with a resultant phase which lowers the probability of finding it there. Thus, to a first approximation, resonance will occur from backscattering from the $i^{\text {th }}$ shell of neighbours when:

$$
2 k \alpha_{i} a+\phi(k)=(2 n+1) \pi
$$

where $n$ is an integer, $k$ is the wave vector of the ejected electron, $\alpha_{i}$ is the distance to the $i^{\text {th }}$ shell of neighbours in units of the lattice parameter, $a$, and $\phi(k)$ is a phase shift which takes into account the effects of travelling through the potential of the atoms and the scattering process itself. Expressing $k$ in terms of the kinetic energy of the ejected electron (i.e. its energy above the threshold), $E$, gives:

$$
E=\frac{\hbar^{2}}{8 m} \frac{((2 n+1) \pi-\phi(k))^{2}}{\alpha_{i}^{2}} \frac{1}{a^{2}}
$$

where $\hbar$ is Planck's constant divided by $2 \pi$ and $m$ is the rest mass of the electron.

Figure 3 shows plots of $E$ versus $1 / a^{2}$ for each of the features, taking the position of the third feature in the VC spectrum at 3 rather than $3^{\prime}$. It is clear that all of the features vary systematically with lattice parameter. Fitting straight lines to points gives three types of behaviour. Peaks 5 and 6 give lines of positive slope passing through the origin as predicted by the simple argument above. Peaks 4 and 7 give lines of positive slope with positive intercepts on the energy axis. Peaks 1,2 and 3 have negative slopes.

Interpreting the slopes of the lines corresponding to peaks 5 and 6 in terms of the above analysis yields values of $2 \pi \alpha_{i} /((2 n+1) \pi-\phi(k))$ of $0.334 \pm 0.015$ and $0.24 \pm 0.01$ respectively. If it 


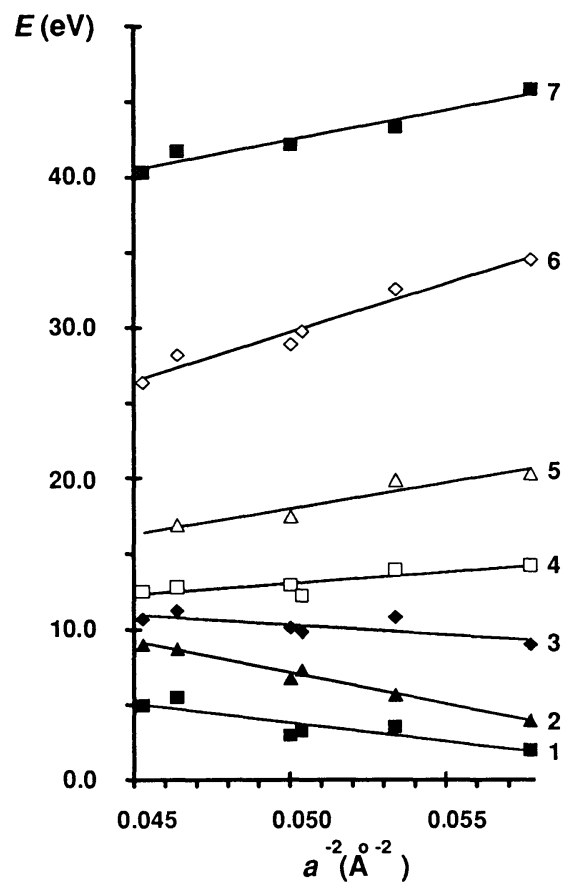

Fig. 3. - A graph of the energy, $E$, of the features labelled in Figure 1 versus $a^{-2}$, where $a$ is the lattice parameter of the compound.

is assumed that each resonance corresponds to the same $n$ and that $\phi(k)$ is similar in the two cases, then the ratio of these values is the ratio of the $\alpha_{i}$ 's involved. Within experimental error, $0.334: 0.240$ is equal to $\sqrt{2}: 1$, the ratio of the distances to the 2 nd and 1 st shells of neighbours in this structure, suggesting that the explanation for these peaks is related to these shells. The round trip distance between the carbon atom and the shell of neighbours can be expressed in terms of the wavelength, $\lambda$, of an electron with an energy equal to that of the resonance peak. For the first shell, corresponding to peak 6 , the average distance is $1.996 \lambda$ and for the second shell, corresponding to peak 5 , the average distance is $2.196 \lambda$. In each case, the values for all six compounds lie within $2 \%$ of the average. Thus a similar value of $\phi(k)$ is indeed required in the two cases although there is a clear difference between the exact values. Further, the intensities of peaks 5 and 6 behave in a manner consistent with this explanation. The second shell is composed of carbon atoms and so one would expect peak 5 to be relatively weak and its intensity to be relatively constant whereas the first shell is composed of metal atoms and so one would expect it to be stronger and increase in intensity as the atomic number of the metal atom increases. Such behaviour can indeed be seen in the spectra presented. The other features do not follow the simple analysis given above and require a more detailed treatment beyond the scope of this paper.

As noted above, it is possible to take the position of the third peak in the VC spectrum at $3^{\prime}$. If this is done, the variation of the positions of peaks 2 and 3 vary less systematically with lattice parameter. However, if the labels on peaks 2 and 3 of the $\mathrm{ZrC}$ and $\mathrm{HfC}$ spectra are also exchanged, the variation becomes more systematic. Either better experimental data or a better theoretical understanding is required to determine the position of peak 3 in $\mathrm{VC}$ and to reconcile the differences in shape in this energy region between the spectrum presented here and that presented by Fink [3]. 
Looking at the threshold energies in more detail, it is clear that they are similar within a given group but that those of the group IVA compounds are $\sim 1 \mathrm{eV}$ less than the equivalent group VA compounds. Table II compares the threshold values found in this work with those given by Pflüger et al. [2, 4] and the binding energies of Ramqvist et al. [5] as quoted in reference [6]. It is clear that there is some variation in the values obtained and that there is no systematic trend in the differences. Given the difference in the technique and the energy resolution, it is not too surprising that the ESCA values differ from the EELS values. Both EELS techniques give good agreement for the energy of the $\pi^{*}$ peak of graphite. The same definition of the threshold energy in the carbides appears to have been used in both sets of EELS work and they have comparable energy resolutions. However, the material used were prepared in different ways. Fink's group prepared material by reactive high frequency sputtering onto molybdenum substrates [2]. The films were removed from the substrate by floating onto aqueous $\mathrm{FeCl}_{3}$ solution. In the present work, problems associated with powder materials were encountered but were overcome as described in section 2.4. Pflüger et al. [2] show that there is little difference in the threshold energy in $\mathrm{TiC}_{x}$ as $x$ varies from 0.6 to 1.0. Thus it is unlikely that small differences in stoichiometry account for the differences observed in the threshold energies. (It is interesting to note here that the change of edge shape with stoichiometry shown in reference [2] is also seen as radiation damage proceeds in TiC. Thus, not only does the edge shape change but there is also loss of carbon from the irradiated volume as reported for VC [1].) If it was the material preparation route that caused the observed differences, then it is likely to be an impurity problem. If it was not the material itself, then the answer probably lies in a subtle instrumental artefact.

Table II. - Comparison of the edge threshold energies obtained in this work with those of Pflüger et al. [2, 4]. The energy of the $\pi^{*}$ peak in graphite obtained by Fink [3] is also compared to that found here. For comparison, the carbon 1s binding energies, determined using ESCA by Ramqvist et al. [5] and quoted in reference [6], are also given.

\begin{tabular}{|c|c|c|c|}
\hline & \multicolumn{3}{|c|}{ Threshold Energy (eV) } \\
\hline & This work & Pflüger et al [2,4] & $\begin{array}{c}\text { Ramqvist et al [5] } \\
\text { as quoted in ref [6] }\end{array}$ \\
\hline TiC & $281.2 \pm 0.1$ & 281.5 & 281.6 \\
\hline $\mathrm{ZrC}$ & $281.4 \pm 0.2$ & & 281.1 \\
\hline $\mathrm{HfC}$ & $281.4 \pm 0.2$ & & 280.8 \\
\hline $\mathrm{VC}$ & $282.0 \pm 0.1$ & 282.6 & 282.2 \\
\hline $\mathrm{NbC}$ & $282.5 \pm 0.1$ & 282.5 & 281.9 \\
\hline $\mathrm{TaC}$ & $282.2 \pm 0.2$ & & 281.9 \\
\hline Graphite $\pi^{*}$ peak & $285.0 \pm 0.1$ & 285 & $284.3 / 284.5$ \\
\hline
\end{tabular}

\section{Conclusions}

The systematic changes in the carbon K-edges from the six compounds reflect the increase in the number of electrons in the system and the associated change in lattice parameter. On moving from group IVA to group VA compounds, the threshold energy and threshold intensity are increased while the absolute energy of the first peak decreases. Within a group, the effect of the increased number of electrons on going from the $3 \mathrm{~d}$ to the $5 \mathrm{~d}$ transition metals is reflected in the decrease of the height of the first peak, the increase of its width and its shift to higher energy, both in absolute 
terms and relative to the threshold. The peak positions correlate strongly with the metal atom size as reflected in the lattice parameter of the compound. Using a simple model, peaks 6 and 5 can be identified with backscattering from the first and second neighbour shell respectively but the origin of the other peaks needs a more detailed treatment.

While these edge shapes are a useful fingerprint for the binary carbides, they may also offer some help in the case of the mixed metal carbides and nitrides so often found in practice. If these precipitates are single phase and isolated, electron diffraction and elemental analysis using EELS and/or energy dispersive X-ray spectroscopy are normally adequate to characterise them. However, in the case of complex precipitates, it is often difficult to get an unambiguous interpretation and the fine structure is one more source of information. One example where it offers some useful information is the case of "NbC" which has grown on an existing "TiN" precipitate in a microalloyed steel [16]. It is possible to analyse regions of the "NbC" without having underlying "TiN" but very difficult to analyse the "TiN" in the absence of "NbC". Hence it is difficult to determine the degree of inter-mixing of the elements between the two phases. The carbon and nitrogen shapes will reflect the presence of such intermixing of the elements.

\section{Acknowledgements}

The authors would like to acknowledge the support of the Engineering and Physical Sciences Research Council in the provision of the PEELS system and a post-doctoral research assistantship for one of us (LAJG). They would also like to thank Dr. P. Rez for pointing out the importance of the lattice parameter.

\section{References}

[1] Craven A.J., Cluckie M.M., Duckworth S.P. and Baker T.N., Ultramicroscopy 28 (1989) 330.

[2] Pflüger J., Fink J., Crecelius G., Bohnen K.P. and Winter H., Solid State Comm. 44 (1982) 489.

[3] Fink J., Adv. Electron. Electron Phys. 75 (1989) 121.

[4] Pflüger J., Fink J. and Schwarz K., Solid State Comm. 55 (1985) 675.

[5] Ramqvist L., Hamrin K., Johansson G., Fahlman A. and Nordling C., J. Phys. Chem. Solids 30 (1969) 1835.

[6] Moulder J.F., Strickle W.F., Sobol P.E. and Bomben K.D., in: Handbook of X-ray Photoelectron Spectroscopy, J. Chastain Ed. (Perkin-Elmer Corporation, Eden Prairie, 1992) p. 216.

[7] Krivanek O.L., Paterson J.H. and Poppa H., in Proc. 47th EMSA Meeting, J.W. Bailey Ed. (San Francisco Press, San Francisco, 1989) p. 140.

[8] Garvie L.A.J., Craven A.J. and Brydson R., Am. Mineral. 79 (1994) 411.

[9] Garvie L.A.J. and Craven A.J., J. Phys. Chem. Minerals 21 (1994) 191.

[10] Krivanek O.L. and Paterson J.H., Ultramicroscopy 32 (1990) 313.

[11] Neckel A., Rastl P., Eibler R., Weinberger P. and Schwarz K., J. Phys. C: Solid State Phys. 9 (1976) 579.

[12] Neckel A., Int. J. Quantum Chem. 23 (1983) 1317.

[13] Goldschmidt H.J., in Interstitial Alloys (Butterworths, London, 1967) p. 92.

[14] Durham P.J., Pendry J.B. and Hodges C.H., Comput. Phys. Commun. 25 (1982) 193.

[15] Natoli C.R., in: EXAFS and Near Edge Structure, A. Bianconi, L. Incoccia and S. Stipcich Eds. (Springer Series in Chemical Physics 27, Springer Verlag, Berlin, Germany, 1983) 43.

[16] Keijian H., Garvie L.A.J., Craven A.J. and Baker T.N., in Proceedings of ICEM'94, B. Jouffrey, C. Colliex, J.P. Chevalier, F. Glas, P.W. Hawkes, D. Hernadez-Verdun, J. Schrevel and D. Thomas Eds. (Les Éditions de Physique, Les Ulis, 1994) Vol. I, 665. 